\title{
Evaluación de implantes de norgestomet reutilizados en protocolos de sincronización del estro en vacas Brahman
}

\author{
Evaluation of reused norgestomet implants in estrus \\ synchronization protocols in Brahman cows
}

\author{
Luis Uribe-Velásquez, ${ }^{1 *}$ Ph.D, Adriana Correa-Orozco, ${ }^{2}$ MVZ, Lina Cuartas-Betancurth, ${ }^{1}$ MVZ, \\ Diego Villamizar-Ramirez, ${ }^{1}$ MVZ, Santiago Ángel-Botero, ${ }^{3}$ M.Sc
}

\begin{abstract}
${ }^{1}$ Universidad de Caldas, Departamento de Salud Animal, Grupo de Investigación CIENVET. Manizales, Colombia. 2 Universidad de Caldas, Maestría en Ciencias Veterinarias. Manizales, Colombia. ${ }^{3}$ Corporación Universitaria del Huila, Programa de Medicina Veterinaria y Zootecnia. Neiva, Colombia. *Correspondencia: Ifuribe@ucaldas.edu.co
\end{abstract}

Recibido: Febrero de 2011; Aceptado: Diciembre de 2011.

\section{RESUMEN}

Objetivo. Evaluar las concentraciones de progesterona, la manifestación de estro y las tasas de preñez en vacas Bos indicus sometidas a varios protocolos de sincronización del estro con un implante de norgestomet usado previamente. Materiales y métodos. Sesenta vacas recibieron un implante auricular de norgestomet reutilizado y fueron distribuidas en uno de cuatro protocolos: (1) benzoato de estradiol $(B E)+$ progesterona $\left(P_{4}\right)+$ prostaglandina $F_{2 a}(P G)\left(B E+P_{4}+P G\right) ;(2)$ valerato de estradiol $(\mathrm{VE})+$ norgestomet $(\mathrm{NG})(\mathrm{VE}+\mathrm{NG})$; (3) el mismo protocolo $\mathrm{BE}+\mathrm{P}_{4}+\mathrm{PG}$, asociado con $400 \mathrm{UI}$ de gonadotropina coriónica equina (eCG) $\left(\mathrm{BE}+\mathrm{P}_{4}+\mathrm{PG}+\mathrm{eCG}\right)$; (4) el mismo protocolo $\mathrm{VE}+\mathrm{NG}$, asociado con 400 UI de eCG (VE+NG+eCG). El implante fue removido el día 9, con inseminación artificial (IA) 12 horas después de la detección del estro. La preñez fue diagnosticada 45 días después de la IA. Las muestras de sangre fueron tomadas los días 0,4 y 9 (después de colocar el implante) para el análisis de progesterona por RIA. Resultados. En el día 4, las concentraciones de progesterona fueron menores en vacas tratadas con $B E+P_{4}+P G(0.90 \pm 0.73 \mathrm{ng} / \mathrm{ml} ; \mathrm{p}<0.01)$. Las vacas que recibieron $\mathrm{VE}+\mathrm{NG}+\mathrm{eCG}$ tuvieron mayor concentración de progesterona en plasma $(p<0.05)$ en el día $9(4.72 \pm$ $0.79 \mathrm{ng} / \mathrm{mL}$ ) que otros protocolos. Hubo mayor porcentaje de detección de estro $(p<0.05)$ en vacas tratadas con VE+NG+eCG $(66.7 \%)$. Las tasas de preñez no fueron afectadas por los protocolos $(p>0.05)$. Conclusiones. Los implantes de norgestomet reutilizados fueron eficaces para sincronizar el estro y alcanzar tasas de preñez adecuadas en vacas Brahman.

Palabras clave: eCG, estradiol, ganado de carne, progesterona, prostaglandinas (Fuente: Agrovoc). 


\section{ABSTRACT}

Objective. To evaluate progesterone concentrations, occurrence of estrus and pregnancy rates in Bos indicus cows submitted to several estrous synchronization protocols with previously used Norgestomet implants. Materials and methods. Sixty cows were given a reused Norgestomet ear implant on Day 0 and were assigned to receive one of four protocols: (PROT 1) estradiol benzoate (EB) + progesterone $(\mathrm{P} 4)+$ prostaglandin $\mathrm{F}_{2 a}(\mathrm{PG})\left(\mathrm{EB}+\mathrm{P}_{4}+\mathrm{PG}\right) ;($ PROT 2$)$ estradiol valerate $(\mathrm{EV})+$ norgestomet $(\mathrm{NG})$ $(\mathrm{EV}+\mathrm{NG})$; (PROT 3) same as $\mathrm{EB}+\mathrm{P}_{4}+\mathrm{PG}$ protocol, plus a $400 \mathrm{UI}$ equine chorionic gonadotropin (eCG) $\left(\mathrm{EB}+\mathrm{P}_{4}+\mathrm{PG}+\mathrm{eCG}\right) ;(\mathrm{PROT} 4)$ same as EV+NG protocol, plus a $400 \mathrm{UI}$ eCG (EV+NG+eCG). The implant was removed on Day 9, with artificial insemination (AI) $12 \mathrm{~h}$ after detection of estrus. Pregnancy was diagnosed $45 \mathrm{~d}$ after AI. Blood samples were collected on Day 0, 4 and 9 (after ear implant was placed), for progesterone analysis by RIA. Results. On day 4, progesterone concentrations were lower in cows treated with $\mathrm{EB}+\mathrm{P}_{4}+\mathrm{PG}(0.90 \pm 0.73 \mathrm{ng} / \mathrm{ml} ; \mathrm{p}<0.01)$. Cows receiving $\mathrm{EV}+\mathrm{NG}+\mathrm{eCG}$ had greater plasma progesterone concentration $(p<0.05)$ on Day $9(4.72 \pm 0.79 \mathrm{ng} / \mathrm{ml})$ than other treatments. There were greater $(p<0.05)$ percentages of estrous detection in cows treated with $\mathrm{EV}+\mathrm{NG}+\mathrm{eCG}(66.7 \%)$. Pregnancy rates were not affected by the treatment protocols $(p>0.05)$. Conclusions. Reusing Norgestomet implants was effective for synchronizing estrus and promote satisfactory pregnancy rates in Brahman cows.

Key words: eCG, beef cattle, oestradiol, progesterone, prostaglandins (Source: Agrovoc)

\section{INTRODUCCIÓN}

La mayor parte del ganado bovino está localizado en áreas tropicales, donde las razas Bos indicus predominan debido a su tolerancia a la temperatura, a la humedad alta, y la resistencia a infecciones parasitarias (1). En regiones tropicales, los períodos de anestro posparto prolongados (más de 150 días) son característicos de ganado Bos indicus y cruces $B$. taurus/B. indicus; originando pérdidas económicas debido al fracaso en alcanzar el intervalo deseable de 12 meses entre partos (2). Por lo tanto, el desafío más importante en los hatos de las vacas de carne en amamantamiento, principalmente en ganado Bos indicus, es lograr la fecundación durante el anestro posparto.Por esta razón, los tratamientos que inducen los ciclos de fertilidad y permiten la inseminación artificial a tiempo fijo (IATF), tienen un impacto positivo en hatos de carne (3).

La IATF viene siendo ampliamente utilizada en ganado Bos taurus y Bos indicus, y provee un enfoque organizado para aumentar el uso de inseminación artificial (IA) y mejorar la eficiencia reproductiva en hatos de carne (4). El desarrollo de protocolos que reduzcan los problemas asociados a la sincronización de la ovulación y la IA, proporcionan a los productores de ganado, herramientas para mejorar la genética. Sin embargo, las variables como la alimentación, la composición racial, la condición corporal, el intervalo posparto, el clima y la localización geográfica, pueden afectar el éxito de los protocolos IATF (5). Otras consideraciones a tener en cuenta con los protocolos son los costos, el tiempo previsto para realizar la aplicación de hormonas y la inseminación artificial, así como también la mano de obra requerida (6).
Dentro de los programas de IATF basados en progesterona y estrógenos, se encuentran métodos que aumentan la manifestación de estro y el diámetro del folículo dominante, los cuales son importantes para mejorar la fertilidad de vacas Bos indicus en amamantamiento (4). Se ha reportado que cuando hay altas concentraciones circulantes de progesterona, al retirar el dispositivo intravaginal (CIDR), se reduce el diámetro del folículo al momento de la IATF, la detección del estro y la tasa de sincronización, así como las tasas de concepción y de preñez en novillas pospubertales y en vacas no lactantes Nelore (Bos indicus) (7). Después de sincronizar el reclutamiento de una nueva onda folicular, el segundo paso de un protocolo de sincronización de la ovulación es disminuir las concentraciones de progesterona para el inicio del proestro y el desarrollo del folículo ovulatorio (3). Se ha descrito que la reutilización de los CIDR ayuda a reducir las concentraciones circulantes de progesterona al momento de retirarlo $(3,7,8)$.

Además de las ventajas fisiológicas mencionadas, la posibilidad de la reutilización constituye una alternativa interesante en la reducción de costos de los protocolos que utilizan implantes de norgestomet $(9,10)$ o dispositivos intravaginales de progesterona, y en consecuencia, los hace más accesibles para los productores. Así como se ha investigado la reutilización de dispositivos intravaginales de progesterona en protocolos de sincronización del estro y de la ovulación $(3,7,8,11,12)$, también se ha estudiado la reutilización de implantes de norgestomet. Algunos trabajos de investigación demostraron que no hay diferencias significativas en las tasas 
de preñez entre protocolos de IATF que incluyeron implantes de norgestomet nuevos o reutilizados $(9,10)$.

Con el fin de obtener mayor información acerca del desempeño de los implantes de norgestomet reutilizados, como una alternativa para disminuir el costo de los protocolos de sincronización del estro y para utilizarlo en novillas o en especies (Bos indicus) sensibles a las altas concentraciones de $\mathrm{P}_{4}$ exógena, se desarrolló la presente investigación. El objetivo fue evaluar las concentraciones de progesterona, la manifestación de estro y la tasa de preñez en vacas Brahman, sometidas a cuatro protocolos de sincronización del estro con implantes de norgestomet reutilizados.

\section{MATERIALES Y MÉTODOS}

Localización y animales. El trabajo se realizó en la Hacienda Abastecedora Brahman Internacional, ubicada en Planeta Rica, Departamento de Córdoba, Colombia $\left(8^{\circ} 24^{\prime} 0.49^{\prime \prime} \mathrm{N} / 75^{\circ} 34^{\prime} 54.83^{\prime \prime} \mathrm{O}\right)$, con una altitud de $80 \mathrm{msnm}$, temperatura promedio de $30^{\circ} \mathrm{C}$ y una precipitación de 2000 mm/año.

Se seleccionaron 60 vacas Brahman (Bos indicus) puras multíparas (entre 2 y 4 partos) con amamantamiento restringido y una condición corporal entre 5 y 6 en una escala de 1 a 9 (13). Las vacas fueron mantenidas en una pradera mixta de Urochloa humidicola, Urochloa brizantha, Urochloa decumbens, Urochloa mutica y Urochloa plantaginea con acceso al agua ad libitum y sal mineralizada ( $8 \%$ de fósforo). Además, se suministró un bloque alimenticio de $15 \mathrm{Kg}$ por cada 15 animales (175 a $300 \mathrm{~g} /$ animal/ día), el cual contenía 3.8\% de proteína; $1.7 \%$ de grasa; $6.5 \%$ de fibra; $43.2 \%$ de cenizas; $4.5 \%$ de fósforo; $7.4 \%$ de calcio; $13.7 \%$ de cloruro de sodio; $0.9 \%$ de magnesio y $15.0 \%$ de humedad.

Tratamiento hormonal. Los implantes de norgestomet (Crestar ${ }^{\circledR}$, Intervet Colombia LTDA), un progestágeno, habían sido usados una vez durante 9 días. Posteriormente, fueron lavados con agua destilada, empacados en papel de aluminio y refrigerados a $4^{\circ} \mathrm{C}$ durante dos semanas. Todas las vacas $(n=60)$ recibieron un implante de norgestomet reutilizado, en la oreja vía subcutánea, en el día 0 del protocolo y luego fueron distribuidas en uno de los siguientes tratamientos (Figura 1): 1) vacas que recibieron una inyección intramuscular (im) de $2.0 \mathrm{mg}$ de benzoato de estradiol (BE; $2.0 \mathrm{~mL}$ de Benzoato de estradiol Syntex, Syntex, Argentina) y otra de $25.0 \mathrm{mg}$ de progesterona $\left(\mathrm{P}_{4} ; 1.0 \mathrm{~mL}\right.$ de Gestavec, Vecol, Colombia) en el día 0 y la aplicación im de $150 \mu \mathrm{g}$ de cloprostenol (PG;

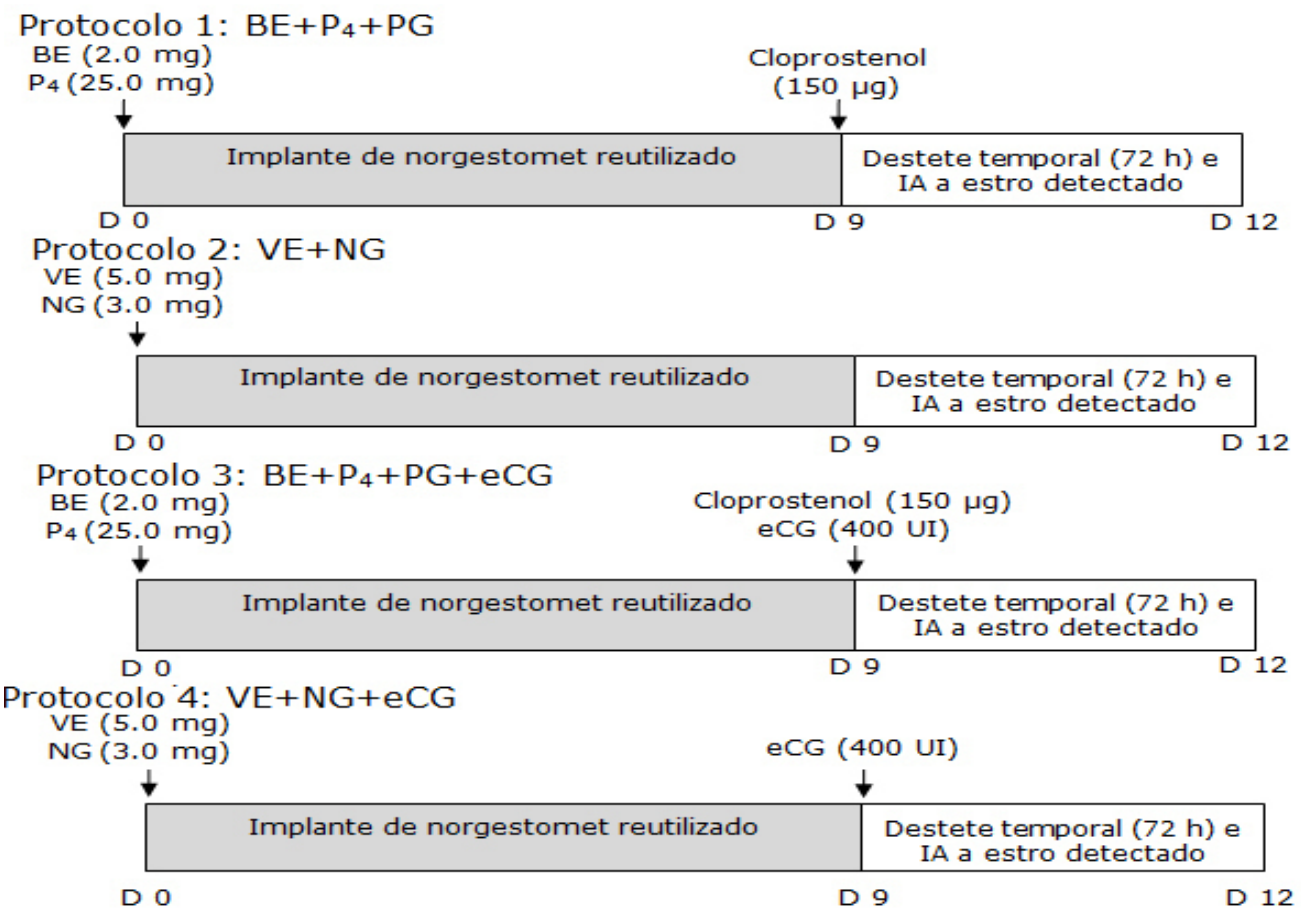

Figura 1. Representación esquemática de los protocolos de sincronización del estro en vacas Brahman, con implantes de norgestomet reutilizados. 
Ciclase $D L$, Syntex, Argentina) el día del retiro del implante (día 9) $\left(B E+P_{4}+P G ; n=15\right) ; 2$ ) vacas que recibieron una inyección im de $5.0 \mathrm{mg}$ de valerato de estradiol y $3.0 \mathrm{mg}$ de norgestomet $\left(\mathrm{VE}+\mathrm{NG} ; 2 \mathrm{~mL}\right.$ Crestar $^{\circledR}$, Intervet Colombia LTDA) en el día $0(V E+N G ; n=15) ; 3$ ) vacas que recibieron el mismo tratamiento 1 , asociado con la aplicación im de 400 UI de gonadotropina coriónica equina (eCG; $2.0 \mathrm{~mL}$ de Folligon, Intervet Colombia LTDA) el día del retiro del implante (día 9) $\left.\left(B E+P_{4}+P G+e C G ; n=15\right) ; 4\right)$ vacas que recibieron el mismo tratamiento 2 , asociado con la aplicación im de 400 UI de eCG el día del retiro del implante (día 9) $(V E+N G+e C G ; n=15)$.

Después de retirar el implante, se realizó destete temporal por 72 horas. La detección de la conducta de estro se realizó de forma visual, teniendo en cuenta signos como el edema vulvar, el flujo cristalino, el reflejo de quietud, la monta activa y la conducta homosexual. La observación empezó 24 horas después de finalizados los protocolos, en la mañana y en la tarde durante una hora cada uno. La IA se realizó 12 horas después de la detección del estro.

Adicionalmente, en el ciclo estral siguiente se realizó observación del estro en todas las vacas del estudio y aquellas que manifestaron estro, fueron inseminadas artificialmente. El diagnóstico de preñez fue realizado por palpación transrectal 45 días después de la IA. Para cada protocolo se analizó la tasa de inseminación, la cual fue calculada al dividir el número vacas inseminadas por el número de vacas tratadas. También se estimaron dos tasas de preñez: la primera fue calculada al dividir el número de vacas preñadas entre el número de vacas inseminadas y la segunda, dividiendo el número de vacas preñadas por el número de vacas tratadas.

Muestras de sangre. Las muestras de sangre fueron colectadas de la vena coccígea en tubo vacutainer ${ }^{\circledR}$ de $10 \mathrm{~mL}$ con heparina los días 0,4 y 9 del protocolo de sincronización, para establecer la concentración de progesterona por radioinmunoanálisis (RIA). Las muestras fueron centrifugadas a $1000 \times \mathrm{g}$ por $10 \mathrm{~min}$ y el plasma conservado a $-20^{\circ} \mathrm{C}$. Posteriormente, las concentraciones plasmáticas de progesterona fueron determinadas mediante kits comerciales (Coat-a-count progesterone; Diagnostic Products Corporation, Los Angeles, CA, EUA) en el Laboratorio de Radioinmunoanálisis de la Universidad Nacional de Colombia, sede Bogotá. La sensibilidad del ensayo fue de $0.02 \mathrm{ng} / \mathrm{ml}$. Todas las muestras fueron procesadas en un solo ensayo.
Análisis estadístico. Las variables dependientes estro a las 48 y 56 horas, tasa de inseminación, proporción de vacas preñadas/hembras inseminadas y proporción de vacas preñadas/ hembras tratadas, la proporción de vacas preñadas/hembras tratadas, se codificaron en una escala binaria de 0 (ausencia) y 1 (presencia), para obtener estimaciones de variabilidad del error estándar. Por tanto, para las variables categóricas y para la concentración plasmática de progesterona, se evalúo el efecto del protocolo, del día, y su interacción lineal, usando el análisis de varianza del programa SAS (14). Las comparaciones múltiples se ajustaron mediante el método de Tukey-Kramer. Con este mismo tipo de análisis, se evalúo el efecto del tipo de estradiol (valerato vs. benzoato) y de progesterona (progesterona vs. norgestomet) utilizados y de la aplicación de prostaglandina y eCG sobre todas las variables dependientes. Los resultados se presentan como medias de mínimos cuadrados \pm el error estándar.

\section{RESULTADOS}

Concentraciones plasmáticas de progesterona. Hubo diferencia significativa en las concentraciones de $\mathrm{P}_{4}$ entre los protocolos $(p<0.0001)$, entre los días $(p<0.05)($ Tabla 1$)$ y en la interacción protocolo y días $(p<0.01)$. En el día 0 del programa de sincronización, los niveles plasmáticos de $\mathrm{P}_{4}$ en el protocolo $\mathrm{BE}+\mathrm{P}_{4}+\mathrm{PG}+\mathrm{eCG}$ fueron más altos en comparación con los otros protocolos $(p<0.01)$. En el día 4 después de colocar el implante de norgestomet, las concentraciones de $P_{4}$ fueron menores $(p<0.01)$ en las vacas del protocolo $B E+P_{4}+P G$. Al momento de retirar el dispositivo (día 9), hubo diferencias significativas en los niveles de $\mathrm{P}_{4}$ del tratamiento $\mathrm{VE}+\mathrm{NG}+\mathrm{eCG}$ en comparación con $\mathrm{BE}+\mathrm{P}_{4}+\mathrm{PG}$ y

Tabla 1. Concentración plasmática de progesterona ( $\mathrm{ng} / \mathrm{mL}$ ) en los días 0,4 y 9 después de colocar el implante reutilizado de norgestomet en vacas Brahman sometidas a sincronización del estro.

\begin{tabular}{|c|c|c|c|}
\hline \multirow{2}{*}{ Protocolo } & \multicolumn{3}{|c|}{$\begin{array}{c}\text { Concentraciones de progesterona } \pm \text { error } \\
\text { estándar }(\mathrm{ng} / \mathrm{mL})\end{array}$} \\
\hline & Día 0 & Día 4 & Día 9 \\
\hline $\mathrm{BE}+\mathrm{P}_{4}+\mathrm{PG}$ & $\begin{aligned} & 2.96 \\
\pm & 0.73 \mathrm{a}, \mathrm{xz}\end{aligned}$ & $\begin{array}{c}0.90 \\
\pm 0.73 \mathrm{a}, \mathrm{yz}\end{array}$ & $\begin{array}{c}1.74 \\
\pm 0.79 \mathrm{a}, \mathrm{xyz}\end{array}$ \\
\hline VE+NG & $\begin{array}{c}2.58 \\
\pm 0.73 \mathrm{a}, \mathrm{xyz}\end{array}$ & $\begin{array}{l}3.51 \\
\pm 0.76 b, x y\end{array}$ & $\begin{array}{c}1.08 \\
\pm 0.73 \mathrm{a}, \mathrm{xz}\end{array}$ \\
\hline $\mathrm{BE}+\mathrm{P}_{4}+\mathrm{PG}+\mathrm{eCG}$ & $\begin{array}{c}6.21 \\
\pm 0.76 \mathrm{~b}, \mathrm{xy}\end{array}$ & $\begin{array}{c}6.24 \\
\pm 0.79 c, x y\end{array}$ & $\begin{array}{c}3.08 \\
\pm 0.76 a, z\end{array}$ \\
\hline VE+NG+eCG & $\begin{array}{c}2.97 \\
\pm 0.73 \mathrm{a}, \mathrm{xz}\end{array}$ & $\begin{array}{c}5.83 \\
\pm 0.73 c, y z\end{array}$ & $\begin{array}{c}4.72 \\
\pm 0.79 b, x y z\end{array}$ \\
\hline
\end{tabular}

BE: Benzoato de estradiol; $P_{4}$ : Progesterona; PG: Prostaglandina; VE: Valerato de estradiol; NG: Norgestomet; eCG: Gonadotropina coriónica equina. $a, b, c$ Medias con letras diferentes en la misma columna son estadísticamente diferentes $(p<0.05$; Tukey-Kramer). $x, y, z$, Medias con letras diferentes en la misma fila son estadísticamente diferentes $(p<0.05 ;$ Tukey-Kramer). x(día 0), y(día 4) y z(día 9). 
$V E+N G(p<0.05)$. No se encontró ningún efecto del tipo de estradiol (BE o VE) o progesterona $\left(P_{4}\right.$ o NG) utilizado sobre las concentraciones de $P_{4}$.

Manifestación del estro. En las primeras 24 horas después de finalizar los protocolos, ninguna vaca mostró signos de estro. La manifestación de estro a las 48 horas fue similar entre protocolos ( $p>0.05)$. El protocolo afectó la presentación de estro a las 56 horas $(p<0.0001)$, en el cual el protocolo $\mathrm{BE}+\mathrm{P}_{4}+\mathrm{PG}+\mathrm{eCG}$ presentó un mayor número de vacas en estro (Tabla 2). La aplicación de eCG en las hembras de los protocolos $B E+P_{4}+P G+e C G$ y VE+NG+eCG $(n=30)$, incrementó la manifestación de estro a las 56 horas $(p<0.0001)$, con una proporción de $9 / 30$ (30.0\%) con eCG y de $1 / 30$ (3.3\%) sin eCG. La presentación de estro a las 48 horas fue menor $(p<0.05)$ en las hembras de los protocolos 1 y $3(n=30)$, las cuales fueron tratadas con $\mathrm{BE}+\mathrm{P}_{4}+\mathrm{PG}(7 / 30 ; 23.3 \%)$ en comparación con las hembras de los protocolos 2 y $4(n=30)$, sincronizadas con VE+NG $(12 / 30 ; 40.0 \%)$.

Tabla 2. Manifestación de estro y tasa de inseminación en vacas Brahman sometidas a sincronización del estro con implante reutilizado de norgestomet.

\begin{tabular}{|c|c|c|c|}
\hline Protocolo & $\begin{array}{c}\text { Estro } 48 \text { h } \\
\text { Proporción } \\
(\%)\end{array}$ & $\begin{array}{c}\text { Estro } 56 \text { h } \\
\text { Proporción } \\
(\%)\end{array}$ & $\begin{array}{c}\text { Tasa de } \\
\text { inseminación }{ }^{1} \\
\text { Proporción } \\
(\%)\end{array}$ \\
\hline $\mathrm{BE}+\mathrm{P}_{4}+\mathrm{PG}$ & $\begin{array}{c}4 / 15 \\
(26.7) a\end{array}$ & $\begin{array}{c}0 / 15 \\
(0.0) a\end{array}$ & $\begin{array}{c}4 / 15 \\
(26.7) \mathrm{a}\end{array}$ \\
\hline VE+NG & $\begin{array}{c}5 / 15 \\
(33.3) a\end{array}$ & $\begin{array}{c}1 / 15 \\
(6.7) \text { ac }\end{array}$ & $\begin{array}{c}6 / 15 \\
(40.0) a b\end{array}$ \\
\hline $\mathrm{BE}+\mathrm{P}_{4}+\mathrm{PG}+\mathrm{eCG}$ & $\begin{array}{c}3 / 15 \\
(20.0) a\end{array}$ & $\begin{array}{c}6 / 15 \\
(40.0) b\end{array}$ & $\begin{array}{c}9 / 15 \\
(60.0) b c\end{array}$ \\
\hline VE+NG+eCG & $\begin{array}{c}7 / 15 \\
(46.7) a\end{array}$ & $\begin{array}{c}3 / 15 \\
(20.0) c\end{array}$ & $\begin{array}{l}10 / 15 \\
(66.7) \mathrm{c}\end{array}$ \\
\hline
\end{tabular}

BE: Benzoato de estradiol; $P_{4}$ : Progesterona; PG: Prostaglandina; VE: Valerato de estradiol; NG: Norgestomet; eCG: Gonadotropina coriónica equina.

Medias con letras diferentes en la misma columna son estadísticamente diferentes $(p<0.05$; Tukey-Kramer)

${ }^{1}$ Proporción de vacas inseminadas comparadas con todas las vacas tratadas

Tasa de inseminación. El protocolo $\mathrm{VE}+\mathrm{NG}+\mathrm{eCG}$ presentó la mayor tasa de inseminación $(\mathrm{p}<0.05)$ (Tabla 2$)$. La aplicación de eCG aumentó el número total de vacas que entraron en estro y fueron inseminadas $(p<0.001)$, con una proporción de 19/30 $(63.3 \%)$, comparado con $10 / 30(33.3 \%)$ de los protocolos sin eCG. Sin embargo, el tipo de estradiol ( $B E \circ \mathrm{VE})$, progesterona $\left(\mathrm{P}_{4} \mathrm{O}\right.$ NG) y la aplicación de PG no afectaron esta proporción $(p>0.05)$.

En el ciclo estral siguiente, la tasa de inseminación no fue influenciada por el protocolo (Tabla 4) ni por el efecto individual de cada hormona empleada $(p>0.05)$.

Tasas de preñez. Entre los protocolos evaluados, no fueron observadas diferencias significativas $(p>0.05)$ en la proporción de vacas preñadas/vacas inseminadas y en la proporción de vacas preñadas/vacas tratadas (Tabla 3). El tipo de estradiol (BE O VE), progesterona ( $N G \circ \mathrm{P}_{4}$ ), la aplicación de $\mathrm{PG}$ y el tratamiento con eCG tampoco afectaron las tasas de preñez $(p>0.05)$.

Tabla 3. Tasas preñez en vacas Brahman sometidas a sincronización del estro con implante reutilizado de norgestomet.

\begin{tabular}{|c|c|c|}
\hline Protocolo & $\begin{array}{l}\text { Vacas preñadas vs } \\
\text { vacas inseminadas } \\
\text { Proporción }(\%)\end{array}$ & $\begin{array}{l}\text { Vacas preñadas } \\
\text { vs vacas tratadas } \\
\text { Proporción (\%) }\end{array}$ \\
\hline $\mathrm{BE}+\mathrm{P}_{4}+\mathrm{PG}$ & $2 / 4(50.0) a$ & $2 / 15(13.3) a$ \\
\hline$V E+N G$ & $4 / 6(66.7) a$ & $4 / 15(26.7) \mathrm{a}$ \\
\hline $\mathrm{BE}+\mathrm{P}_{4}+\mathrm{PG}+\mathrm{eCG}$ & $7 / 9(77.8) a$ & $7 / 15(46.7) \mathrm{a}$ \\
\hline $\mathrm{VE}+\mathrm{NG}+\mathrm{eCG}$ & $6 / 10(60.0) a$ & $6 / 15(40.0) a$ \\
\hline
\end{tabular}

BE: Benzoato de estradiol; $\mathrm{P}_{4}$ : Progesterona; PG: Prostaglandina; VE: Valerato de estradiol; NG: Norgestomet; eCG: Gonadotropina coriónica equina.

Proporciones con letras diferentes en la misma columna son estadísticamente diferentes ( $p<0.05$; Tukey-Kramer)

1 Proporción de vacas preñadas comparadas con las vacas inseminadas

2 Proporción de vacas preñadas comparadas con todas las vacas tratadas

La proporción de vacas preñadas/vacas inseminadas en el siguiente ciclo estral no fue influenciada por el protocolo $(p>0.05)$ (Tabla 4). Sin embargo, la aplicación de eCG si afectó esta proporción $(8 / 9 ; 88.9 \%$ con eCG vs $5 / 8$; $62.5 \%$ sin eCG; $p<0.05)$.

Tabla 4. Tasa de inseminación y tasa de preñez en vacas Brahman en el ciclo estral siguiente a la sincronización del estro con implante reutilizado de norgestomet.

\begin{tabular}{|c|c|c|c|}
\hline Protocolo & $\begin{array}{c}\text { Tasa de } \\
\text { inseminación }{ }^{1} \\
\text { Proporción } \\
(\%)\end{array}$ & $\begin{array}{c}\text { Vacas } \\
\text { preñadas } \\
\text { VS vacas } \\
\text { inseminadas } \\
\text { Proporción } \\
(\%)\end{array}$ & $\begin{array}{c}\text { Vacas que } \\
\text { repitieron } \\
\text { inseminación }\end{array}$ \\
\hline $\mathrm{BE}+\mathrm{P}_{4}+\mathrm{PG}$ & $\begin{array}{c}4 / 15 \\
(26.7) a\end{array}$ & $\begin{array}{c}2 / 4 \\
(50.0) a\end{array}$ & 1 \\
\hline$V E+N G$ & $\begin{array}{c}4 / 15 \\
(26.7) a\end{array}$ & $\begin{array}{c}3 / 4 \\
(75.0) a\end{array}$ & 1 \\
\hline $\mathrm{BE}+\mathrm{P}_{4}+\mathrm{PG}+\mathrm{eCG}$ & $\begin{array}{c}3 / 15 \\
(20.0) a\end{array}$ & $\begin{array}{c}3 / 3 \\
(100.0) a\end{array}$ & 1 \\
\hline $\mathbf{V E}+\mathbf{N G}+\mathbf{e C G}$ & $\begin{array}{c}6 / 15 \\
(40.0) a\end{array}$ & $\begin{array}{c}5 / 6 \\
(83.3) a\end{array}$ & 3 \\
\hline
\end{tabular}

BE: Benzoato de estradiol; $\mathrm{P}_{4}$ : Progesterona; PG: Prostaglandina; VE: Valerato de estradiol; NG: Norgestomet; eCG: Gonadotropina coriónica equina.

Proporciones con letras diferentes en la misma columna son estadísticamente diferentes ( $\mathrm{p}<0.05$; Tukey-Kramer)

${ }^{1}$ Proporción de vacas detectadas en estro e inseminadas comparadas con todas las vacas tratadas

2 Proporción de vacas preñadas comparadas con las vacas inseminadas 


\section{DISCUSIÓN}

Concentraciones plasmáticas de progesterona. Las concentraciones de $\mathrm{P}_{4}$ fueron diferentes entre protocolos, entre días y en la interacción protocolo y días. Las diferencias de esta variable en el día 0 , se explican porque posiblemente las hembras al inicio del experimento no estaban en la misma fase del ciclo estral. La presencia o ausencia de un cuerpo lúteo funcional al momento de insertar un CIDR, determina el nivel de aumento inicial de la concentración plasmática de progesterona (12). En novillas Nelore tratadas con un CIDR usado previamente por 18 días, las concentraciones de progesterona al momento del retiro del implante (día 9) fueron de $2.53 \pm 0.09 \mathrm{ng} / \mathrm{ml}$ (7).

A pesar de las altas concentraciones de progesterona al momento de retirar los implantes, las cuales tienen efectos negativos sobre el diámetro del folículo dominante, la detección de estro, las tasas de concepción y preñez (7), algunas hembras del presente estudio respondieron al protocolo de sincronización y quedaron preñadas. En un experimento previo (7), se concluyó que el tratamiento con eCG al momento de retirar el CIDR, minimizó cualquier efecto negativo de concentraciones de $\mathrm{P}_{4}$ altas en ese día sobre el tamaño del folículo en el momento de la IATF y sobre la tasa de concepción, mediante un aumento en el diámetro del folículo y un incremento en las concentraciones séricas de $\mathrm{P}_{4}$ siete días después de la IATF. Ésta podría ser parte de la explicación acerca de las adecuadas tasas de preñez obtenidas en los protocolos 3 y 4 (Tabla 3 ), los cuales presentaron las concentraciones de $\mathrm{P}_{4}$ más altas al momento de retirar el implante de norgestomet (Tabla 1 ).

Se han encontrado diferencias entre animales, en las concentraciones séricas de progesterona inducidas por CIDRs nuevos o reutilizados, lo cual aparentemente está asociado a una capacidad diferente para metabolizar la $\mathrm{P}_{4}(12,15)$.

Manifestación del estro. En el presente estudio la eCG aumentó el número total de vacas en estro a las 56 horas. En vacas Nelore, también se encontró que la eCG aumentó el porcentaje de vacas detectadas en estro después de sincronizar con CIDR+PG (16). Otros investigadores en Brasil, encontraron que vacas Nelore sometidas a sincronización con el implante de NG de silicona, la inyección de NG+VE y de eCG, presentaron un mayor diámetro del folículo dominante y una tasa de ovulación más alta (1). Un folículo de mayor tamaño puede producir mayor cantidad de estradiol, por lo cual es factible que se genere el pico preovulatorio de
LH y finalmente la ovulación. Estas condiciones aumentan la posibilidad de manifestación de estro. Probablemente, en el presente estudio, las vacas tratadas con eCG presentaron un mayor tamaño del folículo ovulatorio y una tasa de ovulación más alta debido al estimulo de esta gonadotropina, lo cual les permitió presentar una mejor conducta de estro en comparación con las vacas sin eCG.

Contrario a lo encontrado en el presente estudio, en novillas Nelore sincronizadas con CIDR nuevo o de tercer uso, BE, PG, cipionato de estradiol (CE) y con o sin eCG, no se encontró un efecto de la eCG sobre el porcentaje de hembras detectadas en estro en las 48 horas siguientes al retiro del dispositivo (7).

En este experimento, los protocolos que incluyeron VE+NG, presentaron mayor número de hembras que manifestaron estro a las 48 horas en comparación con los protocolos con $\mathrm{BE}+\mathrm{P}_{4}+\mathrm{PG}$. El tratamiento con el implante de NG de $6 \mathrm{mg}$ y la inyección de NG+VE indujo una alta receptividad sexual en vacas Nelore (10/11), pero sólo el 66\% de ellas ovularon (17). Se ha reportado que el uso de VE+NG puede generar manifestación del estro independiente de la actividad ovárica, posiblemente por la sensibilización de la hembra al estrógeno extraovárico y a la presencia de metabolitos derivados del tratamiento; además se menciona inducción de estro en ausencia de ovulación (9). Según Galina y Orihuela (18), las vacas pueden manifestar comportamiento de monta sin un aparente soporte folicular, incluso con un implante de progesterona. Esto puede depender del efecto sobre la emergencia de una nueva onda folicular que tienen los ésteres del estradiol (9).

Otros autores (19), encontraron que un mayor número de vacas Brahman mostraron actividad de monta cuando el VE fue usado con NG (4/6 NG +VE vs. 1/6 NG, respectivamente). Sin embargo, la tasa de ovulación correspondió al $16 \%(1 / 6)$ para ambos tratamientos. Los investigadores mencionan que parece ser que el estrógeno aplicado es necesario para preparar la sincronización del estro y aumentar el número de vacas con manifestación del estro, pero el NG puede ser necesario para que el estro sea acompañado de ovulación. Lo anterior podría explicar parcialmente, la mayor tasa de preñez encontrada cuando se insemina en el estro natural del ciclo siguiente al tratamiento de sincronización (20). La aplicación de estrógenos es una parte necesaria del tratamiento con Norgestomet, para alcanzar el mayor número de animales expresando comportamiento estral, pero puede no ser necesaria para alcanzar las 
tasas de concepción máximas si ésta no mejora las tasas de ovulación (19).

Por otro lado, en un programa de transferencia de embriones con vacas Brangus en México, la cantidad de usos del CIDR (nuevo, usado una o dos veces) no afectó el porcentaje de estro por día (24 a 80 horas post-retiro del CIDR) (12).

Tasa de inseminación. El mayor número de vacas inseminadas se presentó en el protocolo $\mathrm{VE}+\mathrm{NG}+\mathrm{eCG}$. De forma similar, vacas Hereford - Angus tratadas con VE+NG+eCG presentaron alta tasa de estro $(67.86 \% ; 21 / 28)$, en un periodo de observación de 96 horas siguientes al retiro del implante (20). En el presente estudio, hubo un mayor porcentaje de animales manifestando estro y mayor tasa de inseminación en las vacas tratadas con eCG. También las vacas Nelore de primer parto, en amamantamiento y sincronizadas con CIDR+PG o CIDR+PG+eCG, mostraron altos porcentajes de inseminación y preñez en los primeros 4 días (96 horas) de la estación reproductiva en Brasil, cuando fueron tratadas con eCG (16). En vacas Brahman en Australia, sincronizadas con dos aplicaciones de PG o con NG+NV y monitoreadas cada 6 horas para la detección del estro, se encontró que la aplicación de eCG disminuyó la variación en el intervalo de tiempo entre el final del tratamiento y el estro ( $N G+V E+e C G$ : 30.8 a 57.0 horas) en comparación con vacas sin eCG (PG: 41.2 a 155.6 horas; NG+NV: 37.3 a 86.3 horas y NG+PG: 32.0 a 121.0 horas) (21).

Contradictoriamente, en vacas de carne tratadas con el implante de NG de silicona y la inyección de NG+VE, no fue observado efecto de la eCG sobre la manifestación de estro (22). Igualmente, en novillas Nelore sometidas a sincronización del estro con CIDR de primer, segundo o tercer uso y $B E+P G+e C G$, con detección del estro desde el día del retiro del CIDR (día 9) hasta el día 15 del protocolo e IA 12 horas después, no se encontró efecto del CIDR, PG o eCG sobre la tasa de estro (8).

El intervalo de tiempo entre el retiro del implante de NG y la presentación de estro, puede variar entre diferentes estudios, debido a factores como el número de animales estudiados, la edad, el genotipo, las condiciones de cada sistema productivo, la frecuencia de la detección del estro, la duración del tratamiento con progestágeno y a la fase del ciclo estral en el momento de iniciar el tratamiento (21).

La eCG tiene la capacidad de unirse a receptores de LH y FSH y posee una vida media para la fase de eliminación de 121 horas en la vaca (23). El tratamiento con eCG puede causar un mayor crecimiento folicular y proporciona un ambiente endocrino más adecuado durante el proestro (altas concentraciones de $E_{2}$ en sangre) y el diestro (altas concentraciones de $\mathrm{P}_{4}$ en sangre), los cuales son favorables para la fertilidad (16). En este estudio, en los protocolos en los cuales se aplicó eCG (3 y 4), el incremento en la tasa de inseminación se pudo deber, probablemente, a que esta gonadotropina puede estimular el desarrollo del folículo dominante, al aumentar su tasa de crecimiento (1) y su tamaño $(1,7)$; además, este estimulo puede darse por un amplio periodo de tiempo debido a su vida media larga. En consecuencia, se pudo presentar un aumento en las concentraciones de $E_{2}$ que permitieron una mayor manifestación de estro hasta las 56 horas después de la aplicación de esta gonadotropina.

En el ciclo estral siguiente de las vacas del presente experimento, no hubo efecto del protocolo o de las hormonas aplicadas sobre la tasa de vacas inseminadas. Vacas Nelore primíparas en amamantamiento sincronizadas con CIDR+PG o CIDR+PG+eCG, no mostraron efecto de la eCG sobre el porcentaje de vacas inseminadas en los primeros 30 días de la estación reproductiva en Brasil (16).

Tasa de preñez. En este estudio, los protocolos usados y las hormonas aplicadas $\left(B E, V E, P_{4}\right.$, NG, PG o eCG) no afectaron las tasas de preñez. Del mismo modo, en vacas Hereford - Angus (20) y en novillas Nelore (24), tratadas con VE+NG y con dispositivo de progesterona+PG, respectivamente, tampoco fueron encontrados efectos de la eCG en las tasas de concepción y de preñez. Adicionalmente, en vacas Nelore sincronizadas para IATF con dispositivo intravaginal de progesterona+BE+PG+BE+eCG, los investigadores encontraron que la tasa de preñez (vacas preñadas/vacas tratadas) no fue afectada por el tratamiento con eCG (25).

En vacas Nelore sometidas a un protocolo de sincronización de la ovulación con CIDR+BE+PG+CE, los tratamientos con 200 o 400 UI de eCG tampoco mejoraron las tasas de preñez (vacas preñadas/vacas tratadas) en vacas destetadas temporalmente (26). Según los autores, ello indica que el destete temporal probablemente estimuló adecuadamente la secreción de LH de tal manera que, un soporte gonadotrópico adicional pudo no haber sido requerido para el desarrollo folicular final, lo cual podría explicar parcialmente la ausencia de un efecto significativo de la eCG sobre la preñez de las vacas del presente estudio, las cuales fueron sometidas a destete temporal por 72 horas. 
En novillas Nelore sometidas a sincronización del estro con CIDR de primer, segundo o tercer uso y $B E+P G+e C G$, con detección del estro desde el día del retiro del CIDR (día 9) hasta el día 15 del protocolo e IA 12 horas después, no se encontró efecto del CIDR, PG o eCG sobre las tasas de concepción y de preñez (8).

No obstante, otros estudios describen efectos positivos de la eCG sobre la preñez. El tratamiento con eCG mejoró la tasa de preñez en novillas Nelore $(7,27)$, en vacas Nelore no lactantes (7), en vacas Nelore en amamantamiento (1) y en vacas Nelore y Brangus (28).

Sá Filho et al (1), indican que los efectos de la eCG sobre la tasa de preñez después de la IATF en ganado, han sido inconsistentes. Los autores concluyen que los incrementos en la fertilidad inducidos por eCG fueron debidos principalmente a aumentos en las tasas de preñez en vacas posparto no cíclicas o bajo estrés nutricional (baja condición corporal). En esas circunstancias, el tratamiento con eCG al momento de retirar la progesterona puede proveer soporte gonadotrópico adicional, incrementando el crecimiento del folículo preovulatorio.

En un estudio previo con implantes de norgestomet reutilizados (9), se concluyó que los implantes nuevos 0 reutilizados, asociados a $\mathrm{P}_{4}+\mathrm{BE} \circ \mathrm{a}$ NG+VE en un protocolo de IATF, presentaron tasas de preñez similares en vacas Nelore (48.3\% vs. $48.7 \%$, respectivamente). Otros investigadores (10), tampoco determinaron diferencia significativa en la tasa de preñez en vacas Nelore y mestizas con cría que recibieron un implante nuevo (44.0\%), un implante reutilizado $(35.7 \%)$ o dos implantes reutilizados $(32.0 \%)$, asociado a $P_{4}+B E+P G+B E$ en un protocolo de IATF.

Aunque en el presente experimento no se encontraron diferencias estadísticamente significativas entre las tasas de preñez (vacas preñadas/vacas tratadas) de los cuatro protocolos empleados, cabe anotar que, si se observó una diferencia numérica notable entre los protocolos 1 y 3 , con valores de $13.3 \%$ (2/15) y $46.7 \%(7 / 15)$, respectivamente (Tabla 3 ). Estos resultados pueden ser importantes en términos económicos para las empresas ganaderas, porque obtener 7 preñeces de 15 vacas Brahman sincronizadas con $\mathrm{BE}+\mathrm{P}_{4}+\mathrm{PG}+\mathrm{eCG}$, bajo estas condiciones experimentales, es un logro destacable no solo por la dificultad para obtener resultados satisfactorios en programas de sincronización del estro con este tipo de ganado (Bos indicus), sino también por la relación costo beneficio para el sistema de producción.

En este experimento, la proporción de vacas preñadas/vacas inseminadas en el siguiente ciclo estral, no fue influenciada por el protocolo pero si por la aplicación de eCG. Se ha descrito que, vacas Nelore primíparas en amamantamiento sincronizadas con CIDR+PG o CIDR+PG+eCG, no presentaron efecto de la eCG sobre la tasa de concepción (vacas preñadas/vacas inseminadas) entre el día 5 y 30 después de finalizado el protocolo (16). En un estudio con vacas Bos indicus cruzadas en amamantamiento, se demostró que la aplicación de eCG aumentó las concentraciones plasmáticas de progesterona 12 días después de la terminación del tratamiento (29). La progesterona producida durante la fase luteal corta, puede ser necesaria para el establecimiento de la función luteal normal en el siguiente ciclo estral (2). Por tanto, el efecto de la eCG sobre la preñez del siguiente ciclo estral en el presente estudio, pudo estar asociado con la recuperación de la ciclicidad de animales en anestro, al estimular la producción de progesterona y de esta forma producir una fase luteal de duración normal en el ciclo estral siguiente a la aplicación del protocolo de sincronización.

En conclusión, la aplicación de VE+NG aumentó la manifestación de estro a las 48 horas y el tratamiento con eCG incrementó la manifestación de estro y la tasa de inseminación. Sin embargo, las tasas de preñez no fueron afectadas por la eCG, y tampoco por los protocolos utilizados. Por el contrario, se observó un efecto positivo de la aplicación de eCG sobre la preñez de vacas inseminadas en el siguiente ciclo estral. 


\section{REFERENCIAS}

1. Sá Filho MF, Ayres H, Ferreira RM, Marques MO, Reis EL, Silva RCP et al. Equine chorionic gonadotropin and gonadotropinreleasing hormone enhance fertility in a norgestomet-based, timed artificial insemination protocol in suckled Nelore (Bos indicus) cows. Theriogenology 2010a; 73:651-658.

2. Báez G, Grajales H. Anestro posparto en ganado bovino en el trópico. Rev MVZ Córdoba 2009; 14(3):1867-1875.

3. Meneghetti M, Sá Filho OG, Peres RFG, Lamb GC, Vasconcelos JLM. Fixed-time artificial insemination with estradiol and progesterone for Bos indicus cows I: Basis for development of protocols. Theriogenology 2009; 72:179-189.

4. Sá Filho MF, Crespilho AM, Santos JEP, Perry GA, Baruselli OS. Ovarian follicle diameter at timed insemination and estrous response influence likelihood of ovulation and pregnancy after estrous synchronization with progesterone or progestin-based protocols in suckled Bos indicus cows. Anim Reprod Sci 2010b; 120:23-30.

5. Lamb GC, Dahlen CR, Larson JE, Marquezini G, Stevenson JS. Control of the estrous cycle to improve fertility for fixed-time artificial insemination in beef cattle: a review. J Anim Sci 2010; 88:E181-E192.

6. Mihm M. Delayed resumption of cyclicity in postpartum dairy and beef cows. Reprod Dom Anim 1999; 34:277-284.

7. Peres RFG, Claro Júnior I, Sá Filho OG, Nogueira GP, Vasconcelos JLM. Strategies to improve fertility in Bos indicus postpubertal heifers and nonlactating cows submitted to fixed-time artificial insemination. Theriogenology 2009; 72:681-689.

8. Dias CC, Wechsler FS, Day ML, Vasconcelos JLM. Progesterone concentrations, exogenous equine chorionic gonadotropin, and timing of prostaglandin $F_{2 a}$ treatment affect fertility in postpuberal Nelore heifers. Theriogenology 2009; 72:378-385.
9. Almeida $A B$, Bertan $C M$, Rossa LAF, Gaspar PS, Binelli M, Madureira EH. Avaliação da reutilização de implantes auriculares contendo norgestomet associados ao valerato ou ao benzoato de estradiol em vacas Nelore inseminadas em tempo fixo. Braz J Vet Res Anim Sci 2006; 43(4):456-465.

10. Maluf DZ, Pires AV, Susin I, Moreira RJC, Madureira EH, Binelli M. Avaliação da reutilização de implantes contendo progestágenos na taxa de prenhez em vacas de corte. Braz J Vet Res Anim Sci 2010; 47(1):38-46.

11. Colazo MG, Kastelic JP, Whittaker PR, Gavaga QA, Wilde R, Mapletoft RJ. Fertility in beef cattle given a new or previously used CIDR insert and estradiol, with or without progesterone. Anim Reprod Sci 2004; 81:25-34.

12. Solórzano CF, Mendoza JH, Galina C, Villa A, Vera HR, Romo S. Reutilización de un dispositivo liberador de progesterona (CIDR-B) para sincronizar el estro en un programa de transferencia de embriones bovinos. Téc Pecu Méx 2008; 46(2):119-135.

13. Richards M, Spitzer J, Warner M. Effect of varying levels of postpartum nutrition and body condition at calving on subsequent reproductive performance in beef cattle. J Anim Sci 1986; 62:300-306.

14. SAS Institute Inc. $\circledR$ : Guide for Personal computer [programa de ordenador]. Versión 6.12. Cary (NC): SAS Institute IC.; 1985.

15. Sartori R, Bastos MR, Baruselli PS, Gimenes LU, Ereno RL, Barros CM. Phisiologycal differencies and implications to reproductive management of Bos taurus and Bos indicus cattle in a tropical environment. Soc Reprod Fertil Suppl 2010; 67:357-375.

16. Sá Filho OG, Dias CC, Lamb GC, Vasconcelos JLM. Progesterone-based estrous synchronization protocols in non-suckled and suckled primiparous Bos indicus beef cows. Anim Reprod Sci 2010; 119:9-16. 
17. Pinheiro OL, Barros CM, Figueiredo RA, Do Valle ER, Encarnação RO, Padovani CR. Estrous behavior and the estrus-toovulation interval in Nelore cattle (Bos indicus) with natural estrus or estrus induced with prostaglandin $\mathrm{F}_{2 a}$ or norgestomet and estradiol valerate. Theriogenology 1998; 49:667-681.

18. Galina CS, Orihuela A. The detection of estrus in cattle raised under tropical conditions: What we know and what we need to know. Horm Behav 2007; 52:32-38.

19. Solano J, Orihuela A, Galina CS, Montiel F. Sexual behavior of Zebu cattle (Bos indicus) following estrous induction by Syncro-Mate $B$, with or without estrogen injection. Physiol Behav 2000; 71:503-508.

20. Kastelic JP, Olson WO, Martinez M, Cook RB, Mapletoft RJ. Synchronization of estrus in beef cattle with norgestomet and estradiol valerate. Can Vet J 1999; 40:173-178.

21. Cavalieri J, Rubio I, Kinder JE, Entwistle KW, Fitzpatrick LA. Synchronization of estrus and ovulation and associated endocrine changes in Bos indicus cows. Theriogenology 1997; 47:801-814.

22. Duffy $P$, Crowe MA, Austin EJ, Mihm M, Boland MP, Roche JF. The effect of eCG or estradiol at or after norgestomet removal on follicular dynamics, estrus and ovulation in early post-partum beef cows nursing calves. Theriogenology 2004; 61:725-734.

23. Murphy BD, Martinuk SD. Equine chorionic gonadotropin. Endocr Rev 1991;12:1305-1319.

24. Pegorer MF, Ereno RL, Satrapa RA, Pinheiro VG, Trinca LA, Barros CM. Neither plasma progesterone concentrations nor exogenous eCG affects rates of ovulation or pregnancy in fixed-time artificial insemination (FTAI) protocols for puberal Nellore heifers. Theriogenology 2011; 75:17-23.
25. Pinheiro VG, Souza AF, Pegorer MF, Satrapa RA, Ereno RL, Trinca LA et al. Effects of temporary calf removal and eCG on pregnancy rates to timed-insemination in progesterone-treated postpartum Nellore cows. Theriogenology 2009; 71:519-524.

26. Sá Filho OG, Meneghetti M, Peres RFG, Lamb GC, Vasconcelos JLM. Fixed-time artificial insemination with estradiol and progesterone for Bos indicus cows II: Strategies and factors affecting fertility. Theriogenology 2009; 72:210-218.

27. Sá Filho MF, Torres-Júnior JRS, Penteado L, Gimenes LU, Ferreira RM, Ayres $\mathrm{H}$ et al. Equine chorionic gonadotropin improves the efficacy of a progestin-based fixed-time artificial insemination protocol in Nelore (Bos indicus) heifers. Anim Reprod Sci 2010c; 118:182-187.

28. Rossa LAF, Bertan CM, Almeida AB, Gaspar PS, Mazza PH, Binelli M et al. Efeito do eCG ou benzoato de estradiol associado ao norgestomet na taxa de concepção de vacas de corte submetidas à IATF no pós-parto. Braz. J Vet Res Anim Sci 2009; 46(3):199-206.

29. Baruselli PS, Reis EL, Marques MO, Nasser LF, Bo GA. The use of hormonal treatments to improve reproductive performance of anestrous beef cattle in tropical climates. Anim Reprod Sci 2004; 83:479-486. 